\title{
Computational Modeling of Transmission Capacity with Outage Constraints in Ad-Hoc Networks
}

\author{
Moses Ekpenyong \\ Department of Computer Science, University of Uyo \\ PMB. 1017 520001, Uyo, Akwa Ibom State, Nigeria \\ Tel: 23-4-803-793-3961_E-mail: ekpenyong_moses@yahoo.com \\ Joseph Isabona \\ Department of Basic Sciences, Benson Idahosa University \\ PMB. 1100, Benin City, Benin, Nigeria \\ Tel: 23-4-703-918-1006_E-mail: josabone@yahoo.com
}

\begin{abstract}
This paper proposes a transmission capacity model with an overlaid regime that models the cognitive radio technology. We study a case of two coexisting wireless networks (the primary (PR) network and the secondary (SR) network) operating in the same geographical region and sharing the same spectrum and define the transmission capacity as a product of three signal propagation parameters: the density of transmission, the transmission rate and successful transmission probability $(1-$ outage probability $)$. The PR network has a higher priority to access the spectrum, regardless of the SR network, while the SR network limits its interference on the PR network by carefully controlling the density of its transmitters. Using the C++ programming toolkit, we simulate the transmission capacity for both networks and study their tradeoffs in the presence of two propagation constraints: the outage probability and transmission density. Simulation results show that the proposed model minimizes mutual interference by significantly increasing the network spectrum efficiency per unit area as well as the transmission capacity.
\end{abstract}

Keywords: Wireless ad-hoc networks, Outage probability constraints, Network capacity tradeoffs, SINR, Pathloss

\section{Introduction}

Wireless communication networks consist of nodes that communicate with each other over a wireless channel. While some wireless networks have a wired infrastructure of controllers, with nodes connected to the controller over a wireless link, others, such as ad-hoc networks (Ramanathan and Redi, 2002) operate without the benefit of fixed infrastructure. This implies that nodes are responsible for relaying data as well as being sources and links of data. Given these additional responsibilities, it is natural to inquire about the capacity of such networks.

Before a mobile user transmits, it requires channel assignment from a fixed (for cellular networks) or non-fixed (for ad-hoc networks) infrastructure. The mobile radio channel is characterized by rapidly changing channel characteristics. During transmission, it is expected that the signal strength should not drop below a certain threshold (signal strength required for an acceptable communication). At the receiving end, the signal experiences periods of sufficient signal strength or 'non fades' and insufficient signal strength or 'fades'. During period of fades, the user experiences a signal outage (a situation where the signal drops below the noise power level or threshold). The likelihood of an outage occurrence in a certain time fraction is referred to as outage probability. It is the probability that the required signal power of the infrastructure exceed the actual power it gives if a new user is admitted.

The analysis of ad-hoc networks dates back two decades ago. In the 1980's, the term packet radio network was used. Recently, closed-form expressions for ad-hoc network capacity have been discovered (Gupta and Kumar, 2000, Rozovsky and Kumar, 2001, Toumpis and Goldsmith, 2003). The reason for closed-form expressions is due to the difficulty in analyzing ad-hoc networks. This difficulty emanates from the following factors:

(i) Users interfere with each other in a manner that renders modeling difficult

(ii) There is a natural duplex or multiple access scheme

(iii) The distributed nature of the network renders traditional analysis methodologies obsolete.

Although recent research has made great strides towards understanding wireless network capacity, there still 
remain some unanswered fundamental questions. For instance: How does the capacity depend on various system parameters such as channel characteristics, choice of physical layer and power consumption? Also, in recent years, due to the scarcity and poor utilization of the spectrum, the regulatory bodies are beginning to consider the possibility of permitting secondary (SR) networks to coexist with licensed primary (PR) networks. A PR network is a legacy network that has an exclusive right to a certain spectrum band. Examples of PR networks are the common cellular and television broadcast networks. SR networks are responsible for detecting the transmission of PR networks and avoiding interference with them. A SR network user has no spectrum license. Hence additional functionalities are required to share the licensed spectrum band.

The concept of coexistence is the main driving force behind the cognitive radio technology (Haykin, 2005, Neel, 2006, Simeone, Stanojev, Savazzi, Bar-Ness, Spagnolini and Pickholtz, 2008, Xiao and Hu, 2009). In cognitive radio networks, the PR users have a high priority to access the spectrum and the SR users need to operate conservatively such that their interference with the PR users is limited below an "acceptable level". In this overlaid regime, the capacity or throughput scaling laws for the PR and SR networks become interesting problems.

\section{Review of Related Works}

Recent advances in characterizing network capacity were sparked in Gupta and Kumar (2000), with their notion of transport capacity. After their research, a number of studies (Xie and Kumar, 2004, Leveque and Telator, 2005, Ozqur, Leveque and Tse, 2007) have emerged. These studies focus on the behaviour of the end-to-end network capacity, growing within the node limit, under a variety of models of node interaction and fading conditions. The studies also confirm the basic induction in Gupta and Kumar (2000), that, under traditional technological or physical limitations, one node cooperation and signal reception transmission requires an "area" to operate and therefore, the per node end-to-end throughput decays as $\theta\left(\frac{1}{\sqrt{n}}\right)$ for $n$ nodes in the network.

Using percolation theory, Franceschetti, Douse, Tse and Thiran (2007) have established the existence of $\theta(\sqrt{n})$ sum throughput scaling. Therefore, by allowing the nodes to travel independently and uniformly, a constant throughput scaling $\theta(1)$ per S-D pair can be achieved. In Baccelli, Blaszczyszyn and Muhlethaler (2006), a multi-hop spatial reuse ALOHA protocol is proposed by optimizing the product between the number of simultaneous successful transmissions per area and the average transmission range. Here, the transport capacity is proportional to the square root of the node density, which achieves the upper bound of Gupta and Kumar (2000). Weber, Yang, Andrews and de Veciana (2005) have derived the upper and lower bounds of the transmission capacity of spread spectrum wireless ad-hoc networks, where the transmission capacity is defined as the product between the maximum density of successful transmission and the corresponding data rate, under outage probability constraint. All of these results focus on the capacity of a single ad-hoc wireless network. $\mathrm{Vu}$ and Tarokh (2008) considers the throughput scaling law for single-hop cognitive radio network, whose linear scaling law is obtained for the SR network with an outage constraint on the PR network. Jeon, Devroye, Vu, Chung and Tarokh (2009) consider a multi-hop cognitive network on top of a PR network and assume that the SR nodes know the location of each PR node. With a transmission scheme that defines a preservation region around each Pr node, they show that both networks can achieve same throughput scaling law as a stand alone wireless network, while the SR may suffer some finite outage probability. Yin, Gao and Gui (2008) assumes that the SR nodes are familiar with the locations of PR transmitters. They propose a transmission scheme to show that both networks can achieve the same throughput scaling law as a stand-alone wireless network with no outage.

This paper studies the coexistence of two ad-hoc networks with different transmission scales with power and/or transmission range based on the transmission capacity defined in Weber, Yang, Andrew, and de Vaciana (2005). In contrast with $\mathrm{Vu}$ and Tarokh (2008), Joen et.al. (2009), Yin, Gao and Gui (2008), we do not define any preservation region, but resort to a stochastic approach for quantifying the transmission capacities of both the PR and SR networks. We extend the definition of transmission capacity from the single network to overlaid networks through computational modeling. We approach this problem by minimizing the mutual interference effect across two overlaid networks (i.e., the PR network and the SR network). Our aim is to significantly increase the spectrum efficiency (Alouini, 1999) per unit area (a measure of the quantity of users or services that can be simultaneously supported by limited radio frequency bandwidth in a defined geographical area) in wireless ad-hoc networks as well as their transmission capacities and evaluate the outage probability of both the PR network, $P^{0}$, and SR network, $P^{l}$. 


\section{Network Modeling: Assumptions and Preliminaries}

Let the PR and SR networks be ad-hoc networks (i.e. has both transmitting and receiving capabilities). For simplicity, we assume that at a certain time instance, the distribution of PR transmitters (TR) follows a homogenous Poisson point process (PPP), $\mathrm{H}_{0}$, of density $\lambda_{0}$ and the distribution of SR transmitters follow another independent homogenous PPP, $\mathrm{H}_{1}$, of density $\lambda_{1}$. Our aim is to evaluate the outage probability of the PR network, $\mathrm{P}^{\mathrm{o}}$ and that of the SR network $\mathrm{P}^{1}$, which are functions of the TR node densities $\lambda_{0}$ and $\lambda_{1}$.

The network model design is as follows: consider the scenario where a network of PR nodes and a network of SR nodes coexist in the same geographical region, and assume that the PR network is the legacy network, which has a higher priority to access the spectrum. The prerequisite condition for introducing a new SR network into the territory of the PR network is upper-bound limited by a target constraint, $\Delta \in$, where $\Delta \in$ represents the target outage probability increment and usually takes a small value. Similar to Weber et. al. (2005), and in order to evaluate the outage probabilities, we condition a typical PR (or SR) receiver (RS) at the origin, which yields the palm distribution for PR (or SR) TRs. An attempted transmission is successful if the received signal-to-interference-plus-noise ratio (SINR) at the reference RS is above a threshold, $\beta_{j}$, otherwise, the transmission fails, i.e. outage occurs. We use $\beta_{0}$ and $\beta_{1}$ to represent the SINR threshold for the PR network and the SR network respectively.

\subsection{Channel Model}

The channel model is simple. Consider the large-scale pathloss and ignore the effects of shadowing and small multipath fading. The normalized channel power gain $\mathrm{g}(\mathrm{d})$ is given by

$g(d)=k\left(d^{\alpha}\right)^{-1}$

where $k$ is a system-dependent constant, $\mathrm{d}$ is the distance between TR and the corresponding RS and $\alpha>2$ denotes the pathloss exponent. We normalize $k$ to unity and consider a single-hop transmission, assuming that all PR TRs use the same transmission power $P_{0}$, and all PR transmits over the same distance $\gamma_{0}$. Also for simplicity, the ambient noise is assumed to be Additive White Guassian Noise (AWGN) with average power $n$. We assume that all PR TRs and the SR TRs use the same spectrum with bandwidth also normalized to unity. Assuming transmissions at the Shannon target rate is $b=\log _{2}(1+\beta) b p s / H z$, the transmission capacity $C^{\epsilon}$, of a randomly-deployed wireless network, is defined as a product over the maximum density $\lambda^{\epsilon}$ of transmissions, the common transmission data rate $R$ and the probability of successful transmission, $(1-\in)$, with $\in$ having an asymptotically small outage probability. Thus

$C^{\epsilon}=R \lambda^{\epsilon}(1-\epsilon) b p s / H z / M^{2}$

for the maximum density $\lambda^{\epsilon}$ of transmissions. The transmission capacity is then the area spectral efficiency resulting from the maximum density of successful transmissions.

\subsection{Single Network Transmission Capacity}

Let all nodes transmit with the same power $\mathrm{P}_{0}$ and its intended transmitter TR. Assuming that all receivers are located at a fixed distance $\gamma_{0}$ away from their transmitter, the resulting SINR is given by

$$
\operatorname{SINR}=\frac{P_{0} \gamma_{0}^{-\alpha}}{\eta+\sum\left|x_{i}\right|^{-\alpha}}
$$

where $\eta$ is the background noise power, and $\left(x_{i}\right)$ denotes the distance from PR TRs. From equation (3), we derive the asymptotic over vanishingly-small outage probability values for the transmission capacity of the PR network when the SR network is absent. When the SR network is absent, we denote the target outage probability of the PR network over per-link SINR as $\epsilon_{o}$ and obtain

$$
P^{0}=\operatorname{Pr} o b\left(\frac{P_{0} \gamma_{0}^{-\alpha}}{\eta+\sum_{i \in \pi_{0}} P_{0}\left|x_{i}\right|^{-\alpha}} \leq \beta_{0}\right)=\epsilon_{0}
$$


Equation (4) could be re-written as

$\operatorname{Pr} o b\left(X \geq T_{0}\right)=\epsilon_{0}$

where $X=\sum_{i \in \pi_{0}} P_{0}\left|x_{i}\right|^{-\alpha}$ and $T_{0}=\frac{P_{0} \gamma_{0}^{-\alpha}}{\beta_{0}}-\eta$.

The moment generating function (mgf) of $X$ is given in Venkataraman, Haenggi and Collins (2006) as

$\phi_{X}(s)=e^{-\pi \lambda_{0} P_{0}^{2 / \alpha} s^{2 / \alpha} \Gamma\left(1-\frac{2}{\alpha}\right)}$

Considering $\alpha=4$, we have

$\phi_{X}(s)=e^{-\pi^{3 / 2} \lambda_{0} P_{0}^{1 / 2} s^{1 / 2}}$

Through the inverse of Laplace transform, we obtain the probability density function (pdf) of $\mathrm{x}$ as

$f_{X}(x)=\pi / 2 \lambda_{0} \sqrt{P_{0}} x^{3 / 2} e^{\left(-\pi^{3} / 4 x \lambda_{0}^{2} P_{0}\right)}$

and the corresponding cumulative density function (CDF) of $\mathrm{x}$ as

$$
F_{X}(x)=2 Q\left(\frac{\pi^{3 / 2} \lambda_{0} \sqrt{P_{0}}}{\sqrt{2 x}}\right)
$$

From (9), we have

$\operatorname{Pr} o b\left(X \geq T_{0}\right)=1-2 Q\left(\frac{\pi^{3 / 2} \lambda_{0} \sqrt{P_{0}}}{\sqrt{2 T_{0}}}\right)$

Combining equations (5) and (10), it becomes clear that the following condition has to be satisfied:

$Q\left(\frac{\pi^{3 / 2} \lambda_{0} \sqrt{P_{0}}}{\sqrt{2 T_{0}}}\right)=\frac{1-\epsilon_{0}}{2}$

When $\in_{0} \rightarrow 0$, such that $\frac{\pi^{3 / 2} \lambda_{0} \sqrt{P_{0}}}{\sqrt{2 T_{0}}} \rightarrow 0$, we use the Taylor series expansion to obtain the maximum value (via the monotonicity of the $\mathrm{Q}$ function) of $\lambda_{0}$, asymptotically for $\alpha=4$ as

$$
\lambda_{o}^{\epsilon_{o}}=\frac{\epsilon_{0}}{\pi}\left(\frac{T_{0}}{P_{0}}\right)^{1 / 2}=\frac{\epsilon_{0}}{\pi}\left(\frac{\gamma_{0}^{-4}}{\beta_{0}}-\frac{\eta}{P_{0}}\right)^{1 / 2}
$$

As can be observed in equation (12), when the outage probability is negligible, the density of the TRs is a linear function of $\epsilon_{0}$. Therefore, the transmission capacity of the PR network is given by

$$
C_{0}^{\epsilon_{0}}=R_{0} \lambda_{0}^{\epsilon_{0}}\left(1-\epsilon_{0}\right)
$$

where $R_{0}$ represents the data rate when the transmission between the $T_{x}$ and its associated $R_{s}$ is successful, and is set to be same for all the links.

\section{Model Design}

\subsection{Transmission Capacity of the Primary (PR) Network}

When the SR network is present, interference is introduced into the PR network and the outage of the primary network is increased. If we set the target outage probability increment of the PR network as $\Delta \in$, we obtain

$P^{0}=\operatorname{Pr} o b\left(\frac{P_{0} \gamma_{0}^{-\alpha}}{\eta+\sum_{i \in H_{0}} P_{0}\left|X_{i}\right|^{-\alpha}+\sum_{j \in H_{1}} P_{1}\left|Y_{j}\right|^{-\alpha}} \leq \beta_{0}\right)=\epsilon_{0}+\Delta \epsilon$ 
where $Y_{j}$ denote distance from SR TR, to the origin.

With $\gamma=\sum_{j \in H_{1}} P_{1}\left|Y_{j}\right|^{-\alpha}$, equation (14) can be rewritten as

$\operatorname{Pr} o b\left(X+Y \geq T_{0}\right)=\epsilon_{0}+\Delta \in$

The moment generating function (mgf) of $Y$ is given by

$\phi_{Y}(s)=e^{-\pi \lambda_{1} P_{1}^{2 / \alpha} s^{2 / \alpha} \Gamma\left(1-\frac{2}{\alpha}\right)}$

Define $Z=X+Y$, such that the $\operatorname{mgf}$ of $Z$ is given by

$$
\begin{aligned}
\phi_{Z}(s) & =\phi_{X}(s) \phi_{Y}(s) \\
& =e^{-\pi s^{2 / \alpha}\left(1-\frac{2}{\alpha}\right)\left(\lambda_{0} P_{0}^{2 / \alpha}+\lambda_{1} P_{1}^{2 / \alpha}\right)}
\end{aligned}
$$

For $\alpha=4$, we have

$$
\phi_{Z}(s)=e^{-\pi^{1 / 2} s^{1 / 2}\left(\lambda_{0} \sqrt{P_{0}}+\lambda_{1} \sqrt{P_{1}}\right)}
$$

and the pdf of $Z$ is given by

$$
f_{Z}(z)=\frac{\pi}{2}\left(\lambda_{0} \sqrt{P_{0}}+\lambda_{1} \sqrt{P_{1}}\right) Z^{-3 / 2} e^{-\frac{\pi^{3}}{4 z}\left(\lambda_{0} \sqrt{P_{0}}+\lambda_{1} \sqrt{P_{1}}\right)^{2}}
$$

Applying (18) and (15), we have

$$
1-2 Q\left(\frac{\pi^{3 / 2}\left(\lambda_{0} \sqrt{P_{0}}+\lambda_{1} \sqrt{P_{1}}\right)}{\sqrt{2 T_{0}}}\right)=\epsilon_{0}+\Delta \epsilon
$$

which leads us to

$$
2 Q\left(\frac{\pi^{3 / 2}\left(\lambda_{0} \sqrt{P_{0}}+\lambda_{1} \sqrt{P_{1}}\right)}{\sqrt{2 T_{0}}}\right)=\frac{1-\epsilon_{0}-\Delta \epsilon}{2}
$$

When $\in_{0} \rightarrow 0$ and $\Delta \in \rightarrow 0$, a bivariate Taylor series expansion process gives

$$
\frac{1}{2}-\frac{\pi \lambda_{0} \sqrt{P_{0}}}{\sqrt{2 T_{0}}}-\frac{\pi \lambda_{1} \sqrt{P_{1}}}{\sqrt{2 T_{0}}}=\frac{1-\epsilon_{0}-\Delta \epsilon}{2}
$$

Choosing $\lambda_{0}=\lambda_{0}^{\epsilon_{0}}$ as in (12), the maximum allowable value of $\lambda_{1}$ corresponding to the target probability increment $\Delta \in$ is given by

$$
\lambda_{1}^{\Delta \in}=\frac{1}{2}\left(\frac{T_{0}}{P_{1}}\right)^{1 / 2} \Delta \in=\frac{1}{\pi}\left(\frac{P_{0}}{P_{1}} \cdot \frac{\gamma_{0}^{-4}}{\beta_{0}}-\frac{\eta}{P_{1}}\right)^{1 / 2} \Delta \in
$$

and the transmission capacity of the PR network becomes

$$
C_{0}^{\epsilon}=R_{0} \lambda_{0}^{\epsilon_{0}}\left(1-\epsilon_{0}-\Delta \in\right)
$$

Following similar derivations of the PR transmission above, the transmission capacity of the SR network is given by

$$
C_{1}^{\epsilon}=R_{1} \lambda_{1}^{\epsilon}\left(1-\epsilon_{1}\right)
$$

where $R_{1}$ is the data rate adopted by successful SR links. Note the derivation of equation (24) is omitted for 
brevity.

On the other hand, if we simultaneously set the target probability on the PR network as $\epsilon_{0}+\Delta \in$, and set the target outage probability of the SR network as $\epsilon_{1}$, we could choose the value of $\lambda_{1}^{\epsilon}$ in equation (24) as

$$
\lambda_{1}^{\epsilon}=\operatorname{Min}\left(\lambda_{1}^{\Delta \epsilon}, \lambda_{1}^{\epsilon_{1}}\right)
$$

where $\lambda_{1}^{\epsilon_{1}}$ is given by

$$
\lambda_{1}^{\epsilon_{1}}=\frac{\epsilon_{1}}{\pi}\left(\frac{\gamma_{1}^{-2}}{\beta_{1}}-\frac{\eta}{P_{1}}\right)^{1 / 2}-\lambda_{0}^{\epsilon_{0}} \sqrt{\frac{P_{0}}{P_{1}}}
$$

To study the tradeoff of transmission capacities between PR network and SR network, we rewrite equations (23) and (24) as follows

$$
C_{0}^{\epsilon}=R_{0} \lambda_{0}^{\epsilon_{0}}\left(1-\epsilon_{0}-\frac{\pi}{\sqrt{\frac{P_{0} \gamma_{0}^{-\alpha}}{\beta_{0}}-\eta}} \lambda_{1}^{\epsilon} \sqrt{P_{1}}\right)
$$

and

$$
C_{1}^{\epsilon}=R_{1} \lambda_{1}^{\epsilon}\left(1-\frac{\pi \lambda_{0}^{\epsilon_{0}}}{\sqrt{\frac{P_{1}}{P_{0}} \frac{\gamma_{1}^{-\alpha}}{\beta_{1}}-\frac{\eta}{P_{0}}}}+\frac{\pi \lambda_{1}^{\epsilon}}{\sqrt{\frac{\gamma_{1}^{-\alpha}}{\beta_{1}}-\frac{\eta}{P_{1}}}}\right)
$$

From equations (27) and (28), we can easily study the impact of $P_{1}$ on $C_{o}^{\epsilon}$ and $C_{1}^{\epsilon}$ respectively.

\subsection{Model Simulation Input}

The derived model equations were simulated using empirical data gathered from ad-hoc experiments in city scenarios (Weber et. al, 2005, Stepanov and Rothemel, 2006, Souley and Cherkaoui, 2005). Constraint parameter such as outage probabilities were varied based on ideal conditions in urban environment. Table 1 shows the input parameters and their corresponding values, used for the simulation. The simulation results were captured and represented graphically for easy interpretation. The following section interprets the obtained results.

\section{Interpretation of Simulation Results}

Figure 1 is a graph showing the dependence of transmission Capacity (TC) on outage probability in the PR network. As can be seen from this figure, TC is maximized when the outage probability $=0.5$. The reason for this performance is that the TC is adapted to maintain the required signal threshold, rather than impaired, thus eliminating the effect of change.

Figure 2 is a plot of TC vs. outage probability in the SR network. Here, the TC is maximized at 0.4 outage. As shown in this figure, we observe that TC increases monotonically over the outage, since the larger the transmission power is, the larger the value of the SR density. Comparing Figure 1 with Figure 2, we see that the PR has a larger TC than SR. This means that PR has a higher priority to access the spectrum without peculiar contradictions of the SR network.

Figure 3 gives the system performance in terms of the SR density while varying with transmission capacity. Here, as the density of SR radically decreases, the TC increases slowly rather than sharply. This is due to the fact that SR network limits interference to the PR network by carefully controlling the density of the transmitter.

\section{Conclusion}

In this paper, we extended the transmission capacity definition from a single network case to an overlaid network case, which consists of two coexisting networks (the PR and SR networks). We also derived the transmission capacities for these networks by considering the mutual interference effect across the networks and studied through computer simulation, the tradeoffs on both networks. Results obtained show that the PR network has a 
larger transmission capacity than the SR network and if we permit a slight increase in the outage probability of the PR network, the total transmission capacity (i.e. the overall spectrum efficiency per unit area) of the overlaid networks will be boosted significantly over that of a single network.

\section{References}

Alouini, M-S. (1999). Area Spectral Efficiency of Cellular Mobile Radio Systems. IEEE Transactions on Vehicular Technology. 48(4): 1047-1066.

Baccelli, F., Blaszczyszyn, B. and Muhlethaler, P. (2006). An Aloha Protocol for Multihop Mobile Wireless Networks. IEEE Transactions on Information Theory. 52 (2): 421-436.

Franceshetti, M., Dousse, O., Tse, D. and Thiran, P. (2007). Closing the Gap in the Capacity of Wireless Networks via Percolation Theory. IEEE Transactions on Information Theory. 53 (3): 1009-1013.

Gupta, P. and Kumar, P. R. (2000). The Capacity of Wireless Networks. IEEE Transactions on Information Theory. 46 (2): 388-404.

Haykin, S. (2005). The Cognitive Radio: Brain-empowered wireless communications. IEEE Journal on Selected Areas in Communication. 23 (2): 201-220.

Jeon, S. W., Devroye, N., Vu, M., Chung, S. Y. and Tarokh, V. (2009). Cognitive Networks Achieve Throughput Scaling of A Homogeneous Network. In Proceedings of $7^{\text {th }}$ International Conference on Modeling and Optimization in Mobile, Ad Hoc and Wireless Networks: 364-368.

Leveque, O. and Telator, I. E. (2005). Information-Theoretic Upper Bounds on the Capacity of Large Extended Ad-hoc Wireless Networks. IEEE Transactions on Information Theory. 51 (3): 858-865.

Neel, J. O. (2006). Analysis and Design of Cognitive Radio Networks and Distributed Radio Resource Management Algorithms. PhD Thesis, Virginia Polytechnic Institute and State University, Blackburg.

Ozqur, A., Leveque, O. and Tse, D. (2007). Exact Capacity Scaling of Extended Wireless Networks. IEEE International Symposium on Information Theory: 1441-1445.

Ramanathan, R. and Redi, J. (2002). A brief overview of ad-hoc networks: challenges and directions. IEEE Communications Magazine, 40(5): 20-22.

Rozovsky, R. and Kumar, P. (2001). SEEDEX: A MAC protocol for ad-hoc networks. In Proceedings of the 2 nd ACM International Symposium on Mobile Ad-hoc Networking \& Computing, Long Beach, CA: 67 - 75.

Simeone, O., Stanojev, I, Savazzi, Y., Bar-Ness, Y., Spagnolini U. and Pickholtz, R. (2008). Spectrum Leasing to Cooperative Secondary Ad Hoc Networks. IEEE Journal on Selected Areas in Communications. 26(1): 1-11.

Souley, A-K. H. and Cherkaoui, S. (2005). Realistic Urban Scenarios Simulation of Ad Hoc Networks. In Proceedings of $2^{\text {nd }}$ International Conference on Innovations in Information Technology: 1-10.

Stepanov, I. and Rothemel, K. (2006). Simulating Mobile Ad-Hoc Networks in City Scenarios. In Wired/Wireless Internet Communications: Lecture Notes in Computer Science: 1-12.

Toumpis, S. and Goldsmith, A. J. (2003). Capacity Regions for Wireless Ad-hoc Networks. IEEE Transactions on Wireless Communication. 24 (5): 736-748.

$\mathrm{Vu}, \mathrm{M}$. and Tarokh, V. (2008). Scaling Laws of Single-Hop Cognitive Networks. IEEE Transactions on Wireless Communications. 8(8): 4089-4097.

Weber, S. P., Yang, C., Andrews, J. G. and de Veciana, G. (2005). Transmission Capacity of Wireless Ad-hoc Networks with Outage Constraints. IEEE Transactions on Information Theory. 51 (12): 4091-4102.

Xiao, Y. and Hu, F. (2009). Cognitive Radio Networks. Auerback Publications, Tayor and Francis Group, Boca Raton.

Xie, L. and Kumar, P. (2004). A Network Information Theory for Wireless Communication: Scaling Laws and Optimal Operation. IEEE Transactions on Information Theory. 50: 748-767

Yin, C., Gao, L. and Cui, S. (2008). Scaling Laws for Overlaid Wireless Networks: A Cognitive Radio Network vs. A Primary Network. In Proceedings of the IEEE Global Telecommunications Conference: 1-5. 
Table 1. Simulation input parameters

\begin{tabular}{|l|l|}
\hline Parameter & Value \\
\hline Density of SR network $\left(\lambda_{1}\right)$ & 10 \\
\hline Propagation exponent $(\alpha)$ & 2.5 \\
\hline Transmission capacity of PR network $\left(\gamma_{0}\right)$ & 20 \\
\hline Transmission capacity of SR network $\left(\gamma_{1}\right)$ & 5 \\
\hline Average power of ambient noise $(\eta)$ & $103 \mathrm{~dB}$ \\
\hline Target SINR for PR network $\left(\beta_{0}\right)$ & $10 \mathrm{~dB}$ \\
\hline Target SINR for SR network $\left(\beta_{1}\right)$ & $10 \mathrm{~dB}$ \\
\hline Transmission data rate of PR transmitters $\left(R_{0}\right)$ & $16 \mathrm{~kb} / \mathrm{s}$ \\
\hline Transmission data rate for SR transmitters $\left(R_{1}\right)$ & $15 \mathrm{~kb} / \mathrm{s}$ \\
\hline Transmission power for PR transmitters $\left(P_{0}\right)$ & $20 \mathrm{~dB}$ \\
\hline Transmission power for SR transmitters $\left(P_{1}\right)$ & $0.1 \mathrm{~dB}$ \\
\hline Density of PR network $\left(\lambda_{0}\right)$ & $5-25$ \\
\hline Outage probability on PR network $\left(\in_{0}\right)$ & $0-1$ \\
\hline Outage probability on SR network $\left(\epsilon_{1}\right)$ & $0-1$ \\
\hline
\end{tabular}

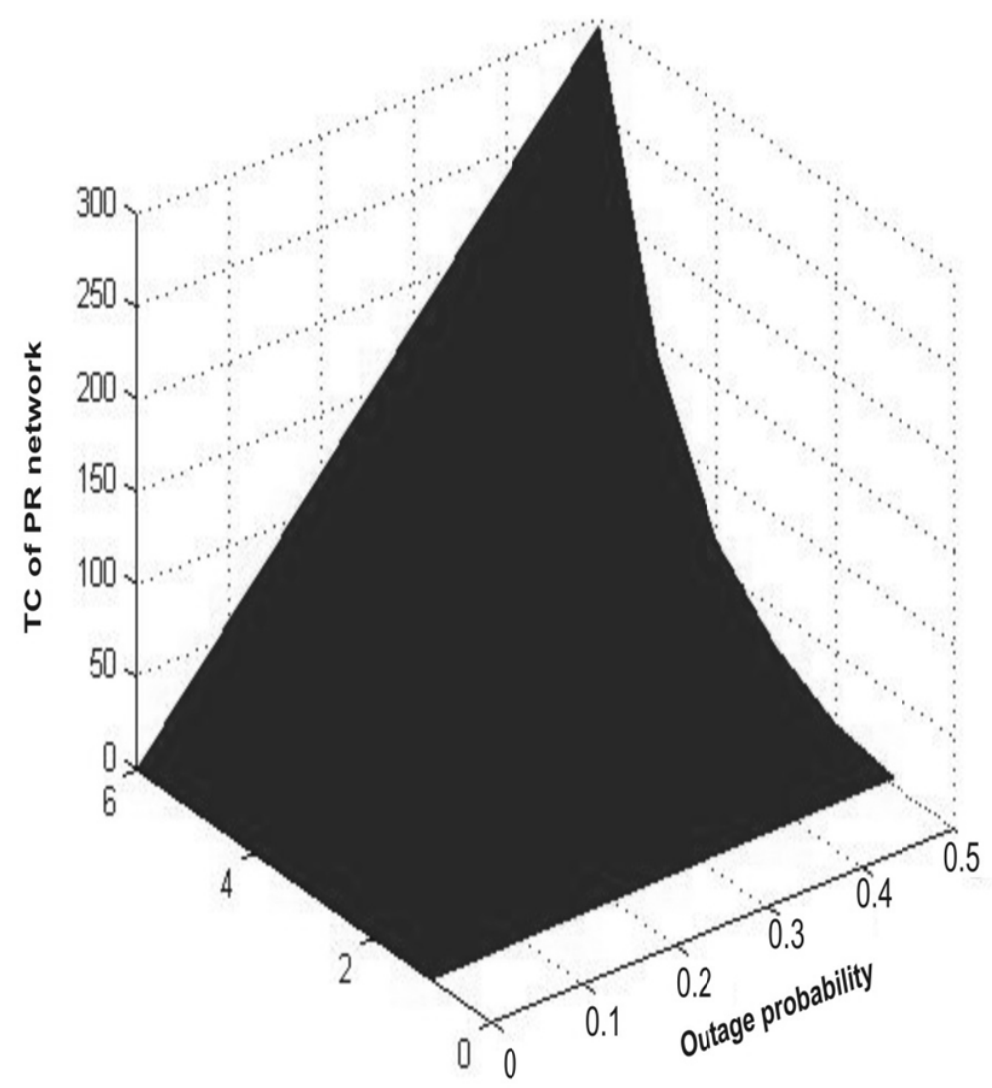

Figure 1. A graph of TC vs. outage for PR networks 


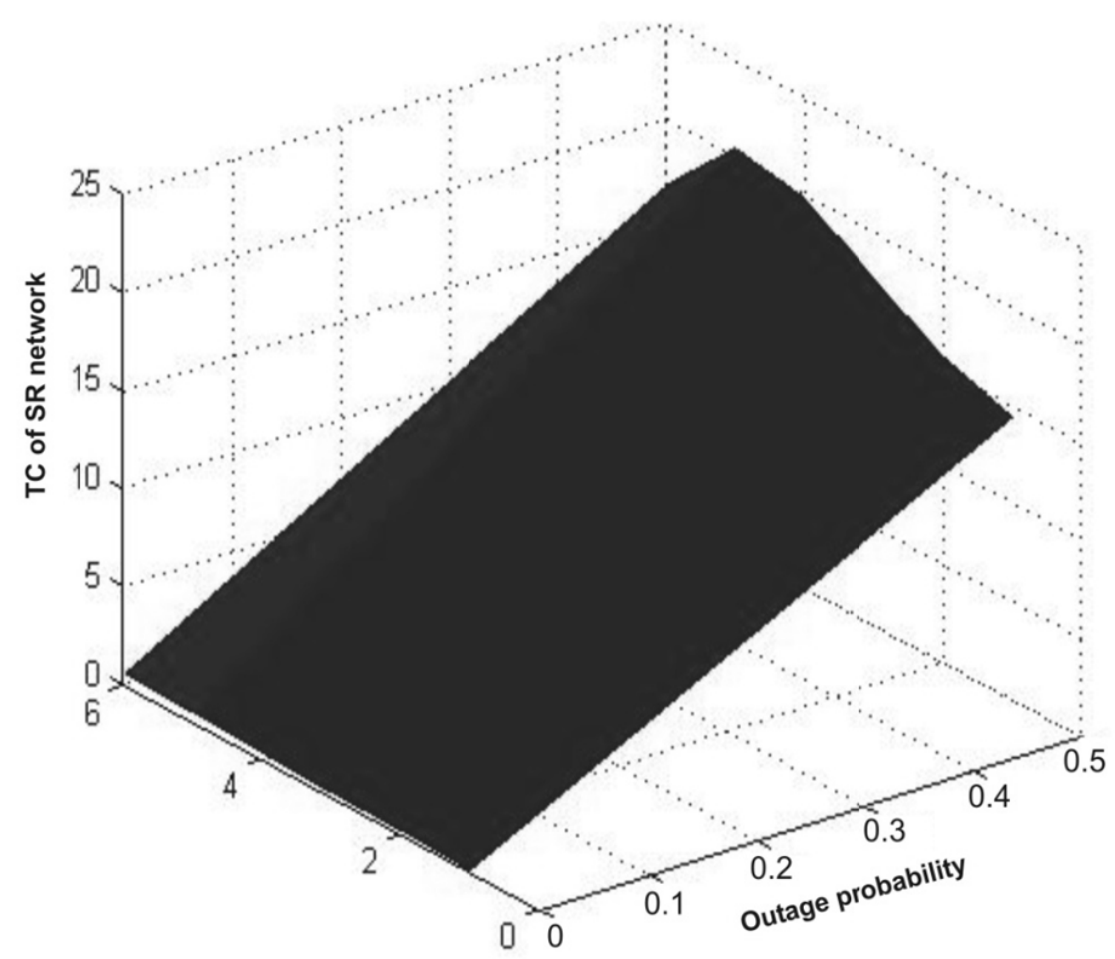

Figure 2. A graph of TC vs. outage for SR networks

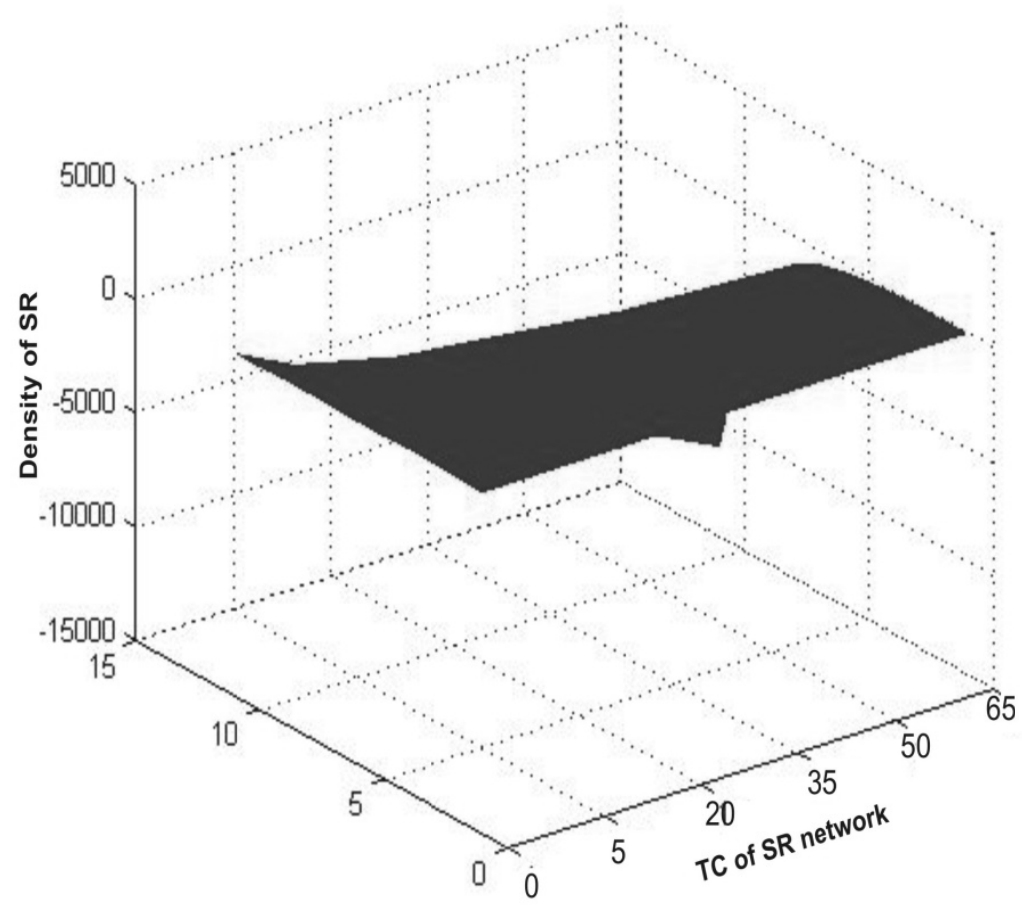

Figure 3. A graph of SR density vs. TC of SR network 